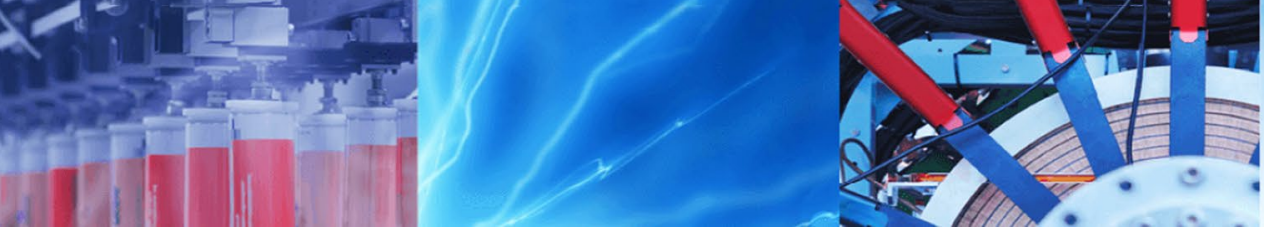

Research Article

\title{
Distributed sliding mode control for formation of multiple nonlinear AVs coupled by uncertain topology
}

\author{
Feng Gao ${ }^{1}\left[\right.$ B Bao Liu ${ }^{1} \cdot$ Jiawei $\mathbf{Q i}^{1} \cdot$ Caimei Wang $^{1}$
}

(c) Springer Nature Switzerland AG 2019

\begin{abstract}
To combat the variety uncertainties in topologies, dynamical models and disturbances, this paper presents a distributed sliding mode control strategy for formation control of multiple AVs. In this scheme, all collected information of each $\mathrm{AV}$ is used for its control and different perturbations are dealt with separately to reduce the contractions among them. Furthermore, a distributed adaptive algorithm is designed to replace the witching part for smoothness of control. The convergence of sliding surfaces of both two controllers is analysed theoretically. The sliding dynamics is affected by both the feedback and interaction topology. The existing decoupling method to handle the variety topologies can be used to synthesize the sliding dynamics. Finally, this approach has been applied to vehicular platooning and validated by several comparative simulations. The results show that the proposed method can control multiple AVs better than the state feedback strategy.
\end{abstract}

Keywords Cooperative control $\cdot$ Sliding mode control $\cdot$ Automated vehicle $\cdot$ Vehicular platoon

\section{Introduction}

A significant amount of studies have been focused on formation control of automatic vehicles (AVs) due to its contributions on traffic performances [1]. Typical strategies include behaviour method, leader-follower method, model prediction method and virtual structure method. The behaviour method was originally proposed for multirobots, and each one runs with some predefined criteria. But it lacks theoretical analysis for global performances. The leader-follower approach was firstly used in PATH program [2]. Recently, some demos have also been performed in the real world $[3,4]$, but it is not widely used because all $A V s$ should receive the information of leader to ensure platoon stability. The model prediction approach focuses on online estimation and rolling optimization to seek the optimal solution [5]. Its theoretical basis is mature, and many problems can be conquered, but the calculation cost is extremely high. The virtual structure approach assumes that there exists a fixed structure among AVs [6].
Except for the behaviour approach, all the aforementioned methods need the global information, which poses great challenges on practical use because of the range limitations of on-board sensors. Moreover, because of the rapid development of wireless communication, it is a trend to apply vehicle-to-vehicle communication (V2V) to AVs, which leads the formation to be a distributed and dynamical network [7]. For simple interaction topologies such as predecessor following type (PFT) and bidirectional type (BDT), many modern control theories have been applied for better performances $[8,9]$ and some special problems have been studied, such as string stability $[9,10]$, homogeneity $[11,12]$ and spacing policies [8]. These approaches use the information of adjacent AVs. For the formation connected by $\mathrm{V} 2 \mathrm{~V}$, the states of more AVs can be collected and intuitively a better performance may be achieved.

If all collected information is used, the connections become complex. A variety of new topologies are generated, which leads to new challenges because there exist time delay and packet loss in wireless communication. For

Feng Gao, gaofeng1@cqu.edu.cn | 'School of Automotive Engineering, Chongqing University, Chongqing, People's Republic of China.

SN Applied Sciences (2019) 1:374 | https://doi.org/10.1007/s42452-019-0395-6 
homogeneous formation interacted by symmetrical topological, Zheng et al. [13] gave out the asymptotic stability condition. In practice, vehicle dynamics are affected by many factors such as vehicle type, environmental resistances and working conditions. To deal with such uncertainties, Gao et al. [14] further presented a robust control method for heterogeneous formations. This synthesis method has been extended to general, but eigenvalue decomposition known topologies in [15] and even with communication delays [16].

Though these synthesis methods can deal with complex topologies, they consider the model uncertainties, nonlinearities, topological variety and disturbances simultaneously, which causes a compromise. To achieve better robust performances, this paper presents a distributed sliding mode control (DSMC) strategy, which has the following advantages:

(1) Comparing with the existing approaches that only use limited information, all signals collected are fed back for control, which may lead to smaller control errors intuitively;

(2) The heterogeneity of AV, model uncertainties and nonlinearities are attenuated by the switching control part. Only the topological variety and disturbance need to be considered when synthesizing the sliding dynamics. A better performance can be achieved by dealing with different requirements separately;

(3) An adaptive algorithm has been further proposed to replace the switching control part to improve driving comfort and lifetime of on-board actuators.

The reminder of this paper is organized as follows: Sect. 2 describes the studied problem. The proposed strategy and the theoretical analysis are introduced in Sect. 3. To validate its effectiveness, the proposed has been applied for vehicular platooning and validated by simulation in Sect. 4, and Sect. 5 concludes the paper.

\section{Problem description}

With approaches such as state/output feedback [17] and decoupling control [18], the motion of AVs in the threedimensional space can be decomposed into six independent one-dimensional motions. To simplify the synthesis process, only one-dimensional motion is considered. The leader of formation is indexed by 0 , and the $N$ followers are indexed by $i=1, \ldots, N$ accordingly. These $N$ heterogeneous AVs are described by the following nonlinear models:

$$
\begin{aligned}
\dot{\boldsymbol{x}}_{i}= & {\left[\begin{array}{ccc}
0 & v_{i}(t) & 0 \\
0 & 0 & a_{i}(t) \\
0 & 0 & f_{i}\left[\mathbf{x}_{i}(t)\right]
\end{array}\right] \boldsymbol{x}_{i}(t) } \\
& +\left[\begin{array}{c}
0 \\
0 \\
g_{i}\left[\boldsymbol{x}_{i}(t)\right]
\end{array}\right] u_{i}(t)+\left[\begin{array}{c}
0 \\
0 \\
\varepsilon_{i}(t)
\end{array}\right], \\
\boldsymbol{x}_{i}(t)= & {\left[\begin{array}{c}
p_{i}(t) \\
v_{i}(t) \\
a_{i}(t)
\end{array}\right], } \\
\left|\varepsilon_{i}(t)\right| \leq & h_{i}\left[\boldsymbol{x}_{i}(t)\right], \quad i=1, \ldots, N,
\end{aligned}
$$

where $p_{i}(t), v_{i}(t), a_{i}(t), u_{i}(t), \varepsilon_{i}(t) \in \mathbb{R}$ are the position, speed, acceleration, control input and disturbance of $A V$ $i$, respectively. Further, $f_{i}(\cdot): \mathbb{R}^{3} \rightarrow \mathbb{R}$ and $g_{i}(\cdot): \mathbb{R}^{3} \rightarrow \mathbb{R}$ are given, which denote their dynamics. The disturbance $\varepsilon_{i}(t)$ may arise from the unmodelled dynamics, parametric errors, environmental disturbances, etc., and $h_{i}(\cdot): \mathbb{R}^{3} \rightarrow \mathbb{R}$ is the upper bound of $\varepsilon_{i}(t)$.

The objective of formation control is to make all followers track the leader as [13]:

$\lim _{t \rightarrow \infty}\left[p_{i}(t)-p_{0}(t)\right]=\delta_{i}$

$\lim _{t \rightarrow \infty}\left[v_{i}(t)-v_{0}(t)\right]=0, \quad i=1, \ldots, N$

where $\delta_{i}$ is a constant. Each AV shares its states with others by V2V [7]. Such information connections are unreliable because wireless communication is easily degraded by adverse environments [19-21]. Moreover, each AV can hardly get the information of all AVs because of the limitation of communication range. The objective of this paper is to propose a DSMC strategy, which can ensure the robust performances of formation in the presence of heterogeneity of AV, uncertainties, disturbances and non-ideal information connections simultaneously.

\subsection{Distributed sliding mode controller design}

Considering the control objective and to make full use of the obtained information, we define the following distributed sliding surfaces:

$s_{i}(t)=a_{i}(t)+K \sum_{k \in \mathbb{N}_{i}}\left[\begin{array}{l}p_{i}(t)-p_{k}(t) \\ v_{i}(t)-v_{k}(t)\end{array}\right], \quad i=1, \ldots, N$

where $\boldsymbol{K} \in \mathbb{R}^{2}$ is the coefficient to be designed according to the required sliding motion and $\mathbb{N}_{i}$ is a set composed of the index of $A V s k \in\{0, \ldots, N\}$ which communicate with $\mathrm{AV} i$. Considering both the speed and smoothness of convergence, the exponential reaching law is selected [22]:

$\dot{s}_{i}(t)=-\lambda s_{i}(t)$ 
where $\gamma>0 \in \mathbb{R}$ determines the exponential convergence speed of $s_{i}(t)$. A faster convergence speed can be achieved with a bigger $\gamma$. Substituting (1) and (3) into (4), we get the following equivalent control input: formation controlled by the following DASMC converges to zero asymptotically:

$u_{e, i}(t)=-\left\{\lambda s_{i}(t)+f_{i}\left[\boldsymbol{x}_{i}(t)\right] a_{i}(t)+\boldsymbol{K} \sum_{k \in \mathbb{N}_{i}}\left[\begin{array}{l}v_{i}(t)-v_{k}(t) \\ a_{i}(t)-a_{k}(t)\end{array}\right]\right\} / g_{i}\left[\boldsymbol{x}_{i}(t)\right]$

To ensure the convergence of $s_{i}(t)$ in the presence of model uncertainties and external disturbances, a switching control part $u_{s, i}(t)$ is added and the DSMC becomes [22]

$u_{i}(t)=u_{e, i}(t)+u_{s, i}(t)$.

Next the Lyapunov theory is used to design $u_{s, i}(t)$. From (3), the time derivation of $s_{i}(t)$ is

$\dot{s}_{i}(t)=\dot{a}_{i}(t)+\boldsymbol{K} \sum_{k \in \mathbb{N}_{i}}\left[\begin{array}{l}v_{i}(t)-v_{k}(t) \\ a_{i}(t)-a_{k}(t)\end{array}\right]$.

The following equation establishes by combing (1) and (5)-(7) together:

$\dot{s}_{i}(t)=-\lambda s_{i}(t)+g_{i}\left[\mathbf{x}_{i}(t)\right] u_{s, i}(t)+\varepsilon_{i}(t)$

Furthermore, substituting (8) into the time derivation of the Lyapunov function $L_{i}(t)=0.5 s_{i}^{2}(t)$ yields

$\dot{L}_{i}(t)=-\lambda s_{i}^{2}(t)+s_{i}(t) g_{i}\left[\boldsymbol{x}_{i}(t)\right] u_{s, i}(t)+s_{i}(t) \varepsilon_{i}(t)$.

To ensure $L_{i}(t) \leq-\lambda s_{i}^{2}(t)=-2 \lambda L_{i}(t)<0$ when $s_{i}(t) \neq 0$, $u_{s, i}(t)$ is designed to be

$u_{s, i}(t)=-\operatorname{sgn}\left[s_{i}(t) g_{i}\left[\boldsymbol{x}_{i}(t)\right]\right]\left|\frac{h_{i}\left[\boldsymbol{x}_{i}(t)\right]}{g_{i}\left[\boldsymbol{x}_{i}(t)\right]}\right|$.

From the Lyapunov theory, it is known that $s_{i}(t)$ converges to zero with the exponential speed not less than $2 \lambda$.

\subsection{Distributed adaptive sliding mode controller}

Since the switching part in (6) may cause jerks and quick ageing of actuators, an adaptive algorithm is further proposed. For the formation controlled by this new designed distributed adaptive sliding mode controller (DASMC), the following theorem ensures the convergence of the sliding surfaces.

Theorem 1 If the disturbance $\varepsilon_{i}(t)$ can be parameterized by $\varepsilon_{i}(t)=\boldsymbol{\theta}_{i}^{\top} \boldsymbol{w}_{i}(t)$, where $\boldsymbol{\theta}_{i}$ is the unknown but time-invariant parameter and $\boldsymbol{w}_{i}(t)$ is the measurable signal, $s_{i}(t)$ of the $u_{i}(t)=u_{e, i}(t)-\frac{\hat{\boldsymbol{\theta}}_{i}^{\top} \boldsymbol{w}_{i}(t)}{g_{i}\left[\mathbf{x}_{i}(t)\right]}$

$\dot{\hat{\theta}}_{i}(t)=s_{i}(t) \boldsymbol{Q} \boldsymbol{w}_{i}(t)$

where $u_{e, i}(t)$ is described by (5) and $\mathbf{Q}>0$ is designed according to the required adaptive speed.

Proof The acceleration behaviour of AV $i$ is derived by substituting (5) and (11) into (1):

$$
\begin{aligned}
\dot{a}_{i}(t)= & -\lambda s_{i}(t)-\boldsymbol{K} \sum_{k \in \mathbb{N}_{i}}\left[\begin{array}{l}
v_{i}(t)-v_{k}(t) \\
a_{i}(t)-a_{k}(t)
\end{array}\right] \\
& -\left[\hat{\theta}_{i}(t)-\theta_{i}\right]^{\top} \boldsymbol{w}_{i}(t) .
\end{aligned}
$$

Defining the Lyapunov function $L_{i}(t)=0.5 s_{i}^{2}(t)+$ $0.5\left[\hat{\theta}_{i}(t)-\boldsymbol{\theta}_{i}\right]^{\top} \boldsymbol{Q}^{-1}\left[\hat{\boldsymbol{\theta}}_{i}(t)-\boldsymbol{\theta}_{i}\right]$ and substituting (7), (11) and (12) into $\dot{L}_{i}(t)$, we have

$\dot{L}_{i}(t)=-\lambda s_{i}^{2}(t) \leq 0$.

From the Lyapunov theory, $s_{i}(t)$ converges to zero asymptotically. Furthermore, form the adaptive control theory, it is known that the estimated parameters $\hat{\theta}_{i}(t)$ can converge to $\theta_{i}$ if the exciting signal has adequate frequencies [23].

\subsection{Sliding dynamics analysis}

On the sliding surface, the following equation establishes since $s_{i}(t)=0$ :

$a_{i}(t)=-\boldsymbol{K} \sum_{j \in \mathbb{N}_{i}}\left[\begin{array}{c}p_{i}(t)-p_{j}(t) \\ v_{i}(t)-v_{j}(t)\end{array}\right]$

To analyse the dynamics of sliding motion and considering the control objective, we define a new error signal $\boldsymbol{e}_{i}(t)=\left[\begin{array}{c}p_{i}(t)-p_{0}(t) \\ v_{i}(t)-v_{0}(t)\end{array}\right]$, which satisfies

$\dot{\boldsymbol{e}}_{i}(t)=\boldsymbol{A} \boldsymbol{e}_{i}(t)+\boldsymbol{B}\left[a_{i}(t)-a_{0}(t)\right]$. 
where $\boldsymbol{A}=\left[\begin{array}{ll}0 & 1 \\ 0 & 0\end{array}\right]$ and $\boldsymbol{B}=\left[\begin{array}{l}0 \\ 1\end{array}\right]$. Substituting (14) into (15) yields the sliding dynamical function:

$\dot{\boldsymbol{e}}_{i}(t)=\boldsymbol{A} \boldsymbol{e}_{i}(t)-\boldsymbol{B} \boldsymbol{K} \sum_{k \in \mathbb{N}_{i}}\left[\boldsymbol{e}_{i}(t)-\boldsymbol{e}_{k}(t)\right]-\boldsymbol{B} a_{0}(t)$

From (16), it is found that the states of different AVs are coupled together by the information connections. To model various connection topologies among AVs in a consistent way, the graph theory is used to describe it as $\mathcal{G}=\mathcal{L}+\mathcal{P} \in \mathbb{R}^{N \times N}[14,15]$. $\mathcal{L}$ describes the connection between followers and is defined as

$\mathcal{L}=\left[I_{i j}\right] \in \mathbb{R}^{N \times N}, l_{i i}=-\sum_{j=1, j \neq i}^{N} I_{i j}, i=1, \ldots, N$.

And $\mathcal{P}$ represents the directional connection from leader to followers:

$\mathcal{P}=\operatorname{diag}\left[g_{1}, \ldots, g_{N}\right] \in \mathbb{R}^{N \times N}$

where $\operatorname{diag}(\cdot)$ denotes a diagonal matrix with the variables being its elements. $g_{i}(t)=1$, if $A V i$ is connected with the leader; otherwise, $g_{i}(t)=0$. Then, the state space function of sliding dynamics is obtained by combining (16)-(18) together:

$\dot{\boldsymbol{E}}(t)=(\boldsymbol{I} \otimes \boldsymbol{A}+\mathcal{G} \otimes \boldsymbol{B} \boldsymbol{K}) \boldsymbol{E}(t)-(\boldsymbol{I} \otimes \boldsymbol{B}) a_{0}(t)$

where the symbol " $\otimes$ " denotes the Kronecker product, $I \in \mathbb{R}^{N \times N}$ is the identity matrix and $\boldsymbol{E}(t)=\left[\begin{array}{lll}\boldsymbol{e}_{i}(t) & \cdots & \boldsymbol{e}_{i}(t)\end{array}\right]^{\top}$.

The current formation control methods based on theories such as $\boldsymbol{H}_{\infty}$ control $[14,15]$ and adaptive approach [1] should deal with the model uncertainties, disturbances and ensure the tracking performances simultaneously. This causes a trade-off between different performances. It is found from (19) that on the sliding surface, the disturbance $\varepsilon_{i}(t)$ is attenuated by the sliding controller and the tracking performance is only degraded by the variety of topology and acceleration of leader. This implies that the proposed control strategy may achieve a better tracking performance.

Furthermore, the sliding dynamics are influenced by both $\boldsymbol{K}$ and $\mathcal{G}$. Some researches have given out the asymptotical stability condition of (19) [13]. If $\mathcal{G}$ is previously known, many advanced control theories can be used to design $\boldsymbol{K}$, such as optimal theory [17], $\boldsymbol{H}_{\infty}$ control [16] and sparse coprime factorization [24]. To deal with the uncertainty of topological matrix, Gao et al. have given out the condition for robust performance in the $\boldsymbol{H}_{\infty}$ sense based on a decoupling way $[14,15]$. And furthermore, a linear matrix inequality (LMI) approach was presented to place the poles of (19) in the required region for eigenvalue-bounded topologies [25]. If the topological matrix is symmetrical called "undirected topology", Wang et al. have proposed a way to numerically solve $\boldsymbol{K}$ ensuring required disturbance attenuation ability, which needs only the minimum eigenvalue of topological matrix [26].

\section{Application and validation}

To validate the proposed method, it has been applied to vehicular platooning, which is composed of one leader (indexed by 0 ) and twelve followers (indexed by $i$ accordingly):

$$
\begin{aligned}
\dot{p}_{i}(t) & =v_{i}(t) \\
\dot{v}_{i}(t) & =a_{i}(t)=\frac{\left[F_{d, i}(t)-F_{r, i}(t)\right]}{M_{i}} \\
\dot{F}_{d, i}(t) & =\left[u_{i}(t)-F_{d, i}(t)\right] / \tau_{i} \\
F_{r, i}(t) & =\phi_{i}\left[v_{i}(t)+v_{w}\right]^{2}+M_{i} g\left[f_{i} \cos (\rho)+\sin (\rho)\right] \\
i & =1, \ldots, 12
\end{aligned}
$$

where $F_{d, i}(t)$ and $F_{r, i}(t)$ are the driving and resistance force, $M_{i}$ is the vehicle mass, $\phi_{i}$ is the aerodynamic drag coefficient, $g$ is the acceleration due to gravity, $f_{i}$ is the rolling resistance coefficient, $v_{w}$ is the wind speed, $\rho$ is the road slope, $\tau_{j}$ is the time constant of drivetrain dynamics and $u_{i}(t)$ is the control input achieved by on-board actuators. The desired distance between two neighbouring vehicles is $5 \mathrm{~m}$. The leader accelerates/decelerates periodically as shown in Fig. 1.

During testing, the coefficient of rolling resistance and time constant of drivetrain dynamics are set to 0.02 and $0.4 \mathrm{~s}$, respectively. The vehicle parameters are generated before simulation randomly, which distribute in their possible range uniformly [11]:

$$
\begin{aligned}
\bar{M} & =1600+50 \mu, \underline{M}=1600-50 \mu, \\
\bar{\phi} & =0.29+0.001 \mu, \underline{\phi}=0.29+0.001 \mu
\end{aligned}
$$

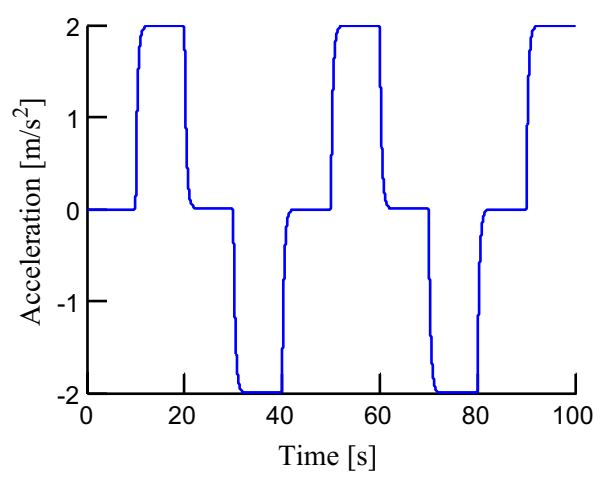

Fig. 1 Acceleration profile of leader 
where $\bar{M}$ and $M$ are the maximum and minimum value of $M_{i}, \bar{\phi}$ and $\phi$ are the maximum and minimum value of $\phi_{i}$ and $\mu$ denotes the uncertain level. The environmental disturbances vary sinusoidally:

$$
\begin{aligned}
v_{w}(t) & =0.4 \mu \sin (\pi t / 4), \\
\rho(p) & =0.01 \mu \sin (\pi p / 200+\pi)
\end{aligned}
$$

where $\pi$ is the circumference ratio and $p$ is the vehicle position.

\subsection{Robust performances}

To evaluate the control effectiveness, the results of DASMC (with the parameters $\gamma=0.3$ and $\boldsymbol{K}=\left[\begin{array}{ll}37.4 & 33.3\end{array}\right]$ ) and
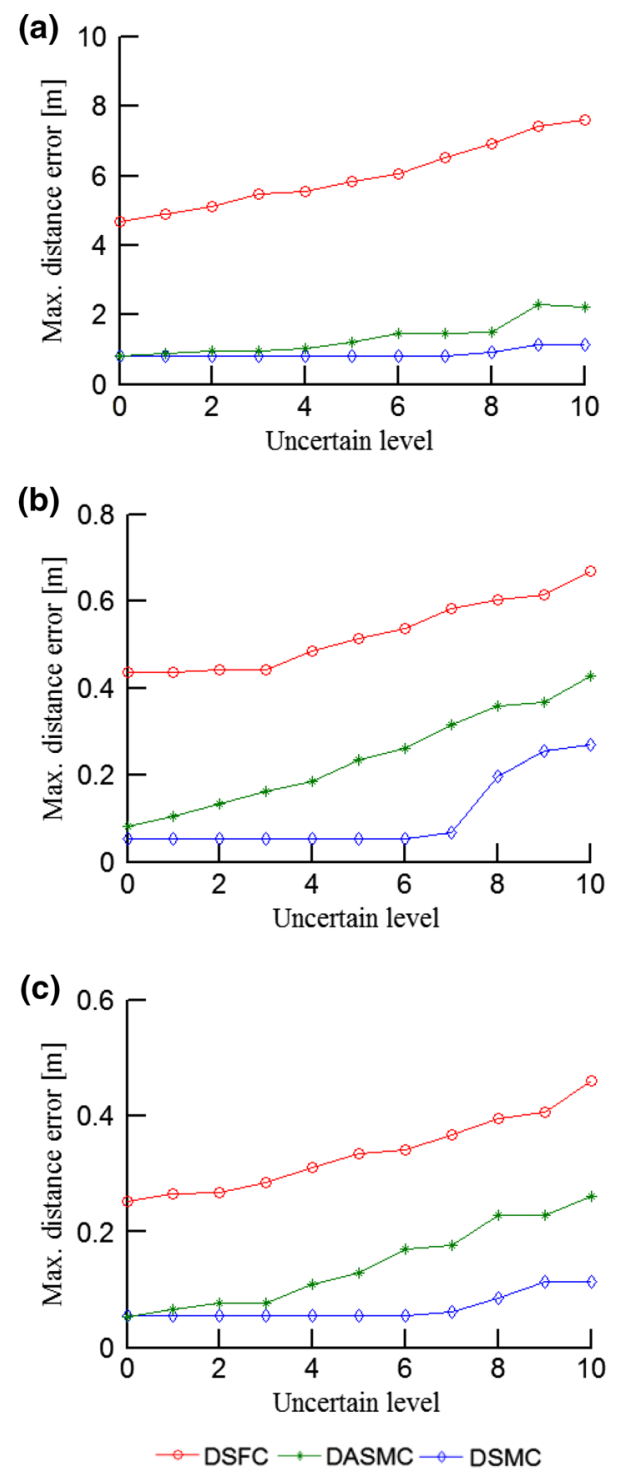

Fig. 2 The maximum distance tracking error. a BDT. b PFT. c TPFT
DSMC have been compared with a distributed state feedback controller (DSFC) designed by using the $\boldsymbol{H}_{\infty}$ approach $[14,15]$ :

$$
\begin{gathered}
F_{d, i}(t)=M_{i, 0} u_{i}(t)+\phi_{i, 0} v_{i}^{2}(t)+M_{i} g f_{i} \\
u_{i}(t)=\boldsymbol{K}_{s} \sum_{k \in \mathbb{N}_{i}}\left[\begin{array}{c}
p_{i}(t)-p_{k}(t)-(i-k) d_{0} \\
v_{i}(t)-v_{k}(t) \\
a_{i}(t)-a_{k}(t)
\end{array}\right], \boldsymbol{K}_{s} \\
=[-8-9-3]
\end{gathered}
$$

where " $\#_{0}$ " represents the nominal value of parameter " $\#$ ". The nonlinearities of powertrain are compensated by (23) firstly and then controlled by (24).

To validate that the proposed method can deal with the uncertainty of topology, the simulations have been conducted with the following topologies [13]: (1) BDT; (2) PFT; (3) two-predecessor following topology (TPFT). The control performances are measured by the maximum tracking error of distance, and the results are shown in Fig. 2.

From the compared results in Fig. 2, it is found that when the uncertain level $\mu \leq 6$, the maximum error of DSMC stays almost the same. Overall, it has the best tracking performance. With the switching control part of DSMC, the parametric errors are attenuated sufficiently. When the
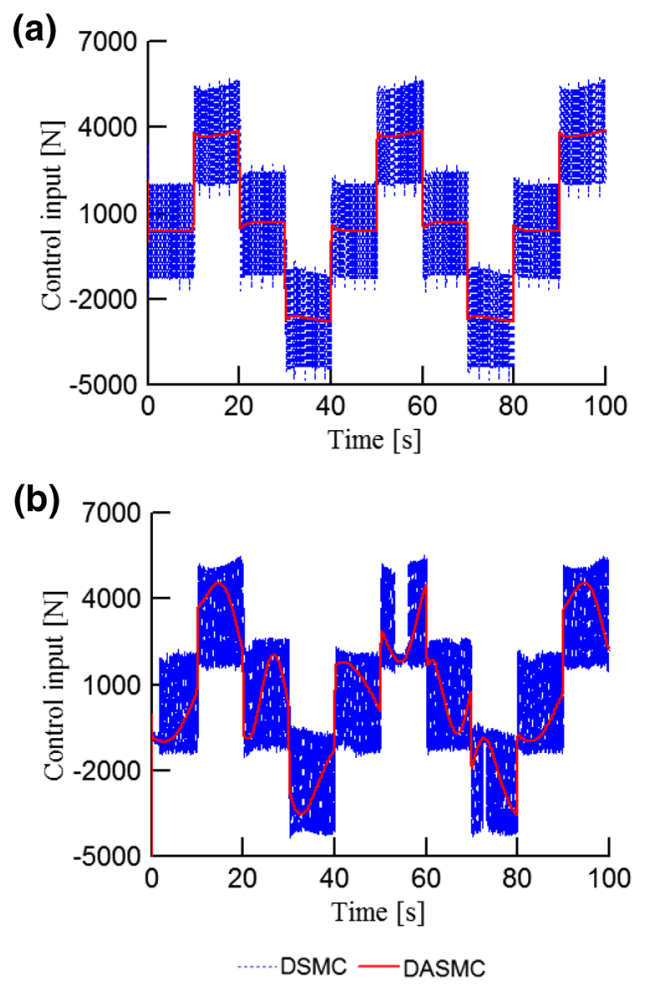

Fig. 3 The control inputs of follower 6 coupled by TPFT as an example. $\mathbf{a} \mu=0 . \mathbf{b} \mu=10$ 
Table 1 Eigenvalue range of topologies

\begin{tabular}{lll}
\hline Topology & $\min _{i=1, \ldots, N} \lambda_{i}$ & $\max _{i=1, \ldots, N} \lambda_{i}$ \\
\hline BDT & 0.0158 & 3.9372 \\
PFT & 1 & 1 \\
TPFT & 1 & 2 \\
\hline
\end{tabular}

uncertain level is sufficiently small, DASMC has almost the same distance attenuation ability as that of DSMC. But its maximum tracking error increases with the uncertain level almost linearly. The reason is that the switching control part is replaced by an adaptive one, which cannot react fast enough, when there exist control errors [23]. It can be concluded from (19) that after the sliding surfaces converge to zero, only the perturbations caused by the leader influence the tracking performance. On the contrary, all kinds of disturbances have to be dealt with when designing the state feedback of DSFC by using the $\mathbf{H}_{\infty}$ control theory $[14,15]$. This causes DSFC to be the worst one under all simulation conditions. If the interaction topology is BDT, the maximum error exceeds $5 \mathrm{~m}$, which implies that a collision happens.

Though DSMC has the best performance, its control input switches with high frequency even when there is no uncertainty (shown in Fig. 3a). This is bad for riding comfort and actuator life. For DASMC, the switching part of SMC is replaced by an adaptive algorithm. Its control input is continuous and smooth, and the adaptive algorithm can adjust the control parameters in good time (shown in Fig. 3b).

Furthermore, Fig. 2 shows that the information topology influences the performance. From (19), it is known that the platoon is composed of multiple subsystems, whose feedback gains depend on the eigenvalues of $\mathcal{G}$. A smaller eigenvalue will reduce the feedback control effect and cause larger error [13]. From Table 1, the minimum eigenvalue of BDT is the smallest, which implies that some of the subsystems have a weak feedback regulation ability. The minimum eigenvalues of PFT and TPFT are the same, but the maximum one of TPFT is bigger. Summarizing the above analysis, the performance of platoon interacted by BDT is the worst and that of TPFT is slightly better than PFT.

\subsection{Random information topology}

In this section, the proposed method is further validated by a random information topology, which is common in practice because of the degradation of wireless communication. A statistical model is used to describe the success possibility $P_{i j}$ of a packet delivery:

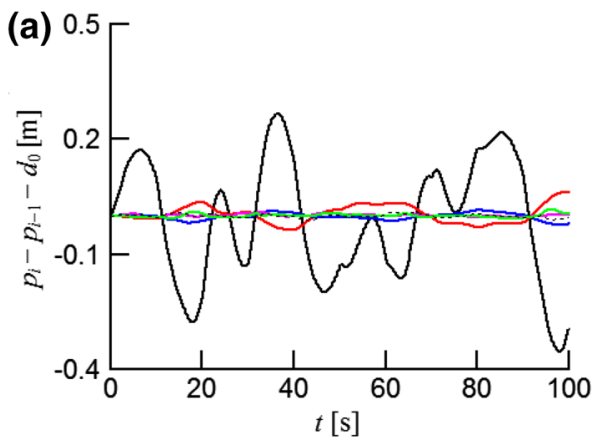

(b)

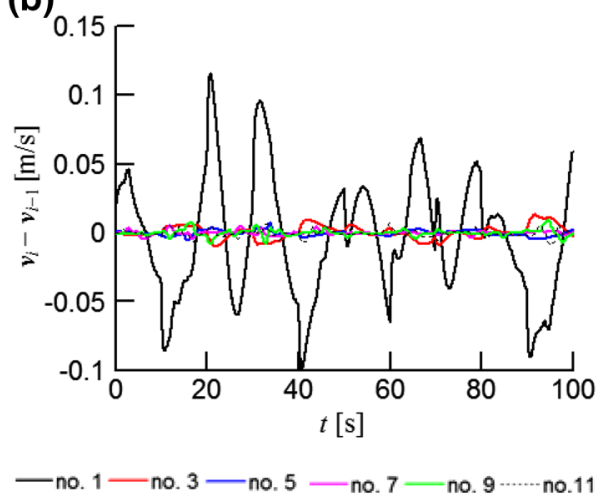

Fig. 4 Results of platoon interacted by a random topology. a Distance tracking error. $\mathbf{b}$ Speed tracking error

$P_{i j}=\left\{\begin{array}{ll}\rho_{i j,} & \text { if } \rho_{i j}>0 \\ 0, & \text { otherwise }\end{array}\right.$,

$\rho_{i j}=-\frac{1}{400}\left|p_{i}-p_{j}\right|^{2}+100$.

If two vehicles are far away, they are difficult to establish an information connection.

During testing, the uncertain level $\mu=10$ and the results are shown in Fig. 4. The maximum control errors of distance and speed are $0.36 \mathrm{~m}$ and $0.12 \mathrm{~m} / \mathrm{s}$, respectively. The topology is assumed to be uncertain, but time invariant, when doing theoretical analysis. It is also applicable for time-varying topologies. Moreover, comparing the errors of different vehicles, it is found that these errors are bounded in a certain range and almost decrease along the platoon. This implies that the disturbance can be dampened along platoon effectively, which is called string stability and needs to be further studied theoretically [9].

\section{Conclusions}

This paper presents a distributed sliding mode control strategy for formation of multiple nonlinear AVs interacted by uncertain topology. Being distinct from state feedback 
strategies, this scheme uses all obtained information and attenuates different perturbations separately. Both theoretical analysis and simulation results demonstrate that:

(1) The proposed method controls multiple AVs with uncertain parameters, disturbed by environmental resistance forces, and interacted by uncertain topologies in a satisfactory manner. It has better robust performance than state feedback control strategy because the variety disturbances are attenuated separately.

(2) The designed adaptive law for uncertain vehicle parameters adjusts the DSMC successfully by using only the collected information of other vehicles. With this adaptive law, the switching part of DSMC is cancelled; meanwhile, the convergence of the sliding surfaces can be guaranteed.

(3) The existing decoupling synthesis method for state feedback can be applied to design the sliding dynamics to attenuate the disturbance arising from acceleration/deceleration of leader.

Acknowledgements This study is supported by National Key R\&D Program of China under Grants 2016YFB0100906 and 2016YFB0101104, Open Fund of State Key Laboratory of Vehicle NVH and Safety under Grant NVHSKL-201705, Industrial Base Enhancement Project under Grant 0714-EMTC02-5593/20.

\section{Compliance with ethical standards}

Conflict of interest The authors declare that they have no conflict of interest.

\section{References}

1. Li R, Zhang L, Han L et al (2017) Multiple vehicle formation control based on robust adaptive control algorithm. IEEE Intell Transp Syst Mag 9(2):41-51

2. Shladover S, Desoer C, Hedrick J et al (1991) Automated vehicle control developments in the PATH program. IEEE Trans Veh Technol 40(1):114-130

3. Tsugawa S, Kao S, Aoki K (2011) An automated truck platoon for energy saving. In: IEEE/RSJ international conference on intelligent robots and systems, San Francisco, pp 4109-4114

4. Chan E, Gilhead P, Jelinek P, et al (2012) Cooperative control of SARTRE automated platoon vehicles. In: Proceedings of 19th ITS World Congress, Vienna, Austria, pp 1-9

5. Ren W, Beard RW, Atkin EM (2007) Information consensus in multi-vehicle cooperative control. IEEE Control System Mag 27(2):71-82

6. Tan KH, Lewis MA (1996) Virtual structure for high precision cooperative mobile robotic control. In: Proceedings of IEEE/RSJ international conference on intelligent robots and systems, pp 132-139
7. Willke T, Tientrakool P, Maxemchuk N (2009) A survey of intervehicle communication protocols and their applications. IEEE Commun Surv Tutor 11(2):3-20

8. Swaroop D, Hedrick J, Chien C et al (1994) A comparison of spacing and headway control laws for automatically controlled vehicles. Veh Syst Dyn 23(8):597-625

9. Liang C, Peng H (1999) Optimal adaptive cruise control with guaranteed string stability. Veh Syst Dyn 31:313-330

10. Guo X, Wang J, Liao F et al (2016) Distributed adaptive integrated sliding mode controller synthesis for string stability of vehicle platoons. IEEE Trans Intell Transp Syst 17(9):2419-2429

11. Li SB, Gao F, Cao D et al (2016) Multiple-model switching control of vehicle longitudinal dynamics for platoon level automation. IEEE Trans Veh Technol 65(6):4480-4492

12. Gao F, Li KQ (2007) Hierarchical switching control of longitudinal acceleration with large uncertainties. Int J Autom Technol 8(3):351-359

13. Zheng Y, Li SE, Wang JQ et al (2016) Stability and scalability of homogeneous vehicular platoon: study on the influence of information flow topologies. IEEE Trans Intell Transp Syst 17(1):14-26

14. Gao F, Dang DD, Huang SS et al (2017) Decoupled robust control of vehicular platoon with identical controller and rigid information flow. Int J Autom Technol 18(1):157-164

15. Xia Q, Gao F, Duan JL et al (2017) Decoupled H-infinity control of automated vehicular platoons with complex interaction topologies. IET Intel Transp Syst 11(2):92-101

16. Gao F, Li SE, Zhen Y et al (2016) Robust control of heterogeneous vehicular platoon with uncertain dynamics and communication delay. IET Intel Transp Syst 10(7):503-513

17. Wang J, Xin M (2013) Integrated optimal formation control of multiple unmanned aerial vehicles. IEEE Trans Control Syst Technol 21(5):1731-1744

18. Su XF, Jia YM, Du JP et al (2015) Modeling and input-output decoupling of hypersonic vehicles. Int J Control Autom Syst 13(1):156-166

19. Oh KK, Park MC, Ahn HS (2015) A survey of multi-agent formation control. Automatica 53(C):424-440

20. Wang Q, Hua QG, Yi Y et al (2017) Multi-agent formation control in switching networks using backstepping design. Int J Control Autom Syst 15(4):1569-1576

21. Wang ZH, Zhang HS, Song XM et al (2017) Consensus problems for discrete-time agents with communication delay. Int J Control Autom Syst 15(4):1515-1523

22. Baek J, Jin M, Han S (2016) A new adaptive sliding mode control scheme for application to robot manipulators. IEEE Trans Ind Electron 63(6):3628-3637

23. Song Y, Zhao K, Krstic M (2017) Adaptive control with exponential regulation in the absence of persistent excitation. IEEE Trans Autom Control 62(5):2589-2596

24. Sabau S, Oara C, Warnick S et al (2017) Optimal distributed control for platooning via sparse coprime factorizations. IEEE Trans Autom Control 62(1):305-320

25. Gao F, Li SE, Chen T, et al (2016) A decoupling method for distributed control of vehicular platoons with V2V. In: Proceedings of IET international conference on intelligent and connected vehicles, Chongqing, China, August, pp 1-6

26. Wang JY, Duan ZS, Wen GH et al (2015) Distributed robust control of uncertain linear multi-agent systems. Int J Robust Nonlinear Control 25(13):2162-2179

Publisher's Note Springer Nature remains neutral with regard to jurisdictional claims in published maps and institutional affiliations. 